\title{
Transcranial Color Coded Duplex Sonography Findings in Stroke Patients Undergoing Rehabilitation: An Observational Study
}

\author{
Srikant Venkatakrishnan ${ }^{1}$ Meeka Khanna ${ }^{1}$ Anupam Gupta ${ }^{1}$ \\ ${ }^{1}$ Department of Neurological Rehabilitation, National Institute of \\ Mental Health and Neurosciences (NIMHANS), Bengaluru, \\ Karnataka, India \\ Address for correspondence Meeka Khanna, Department of \\ Neurological Rehabilitation, National Institute of Mental Health and \\ Neurosciences (NIMHANS), Bengaluru, Karnataka 560029, India \\ (e-mail: meekakhanna@gmail.com).
}

J Neurosci Rural Pract 2022;13:129-133.

\begin{abstract}
Keywords

- rehabilitation outcomes

- stroke

- transcranial colorcoded duplex sonography
\end{abstract}

Background Transcranial color-coded duplex sonography (TCCD) provides information on intracranial blood flow status in stroke patients and can predict rehabilitation outcomes.

Objective This study aimed to assess middle cerebral artery (MCA) parameters using TCCD in MCA territory stroke patients admitted for rehabilitation and correlate with clinical outcome measures.

Materials and Methods Patients aged 18 to 65 years with a first MCA territory stroke, within 6 months of onset were recruited. The clinical outcome scales and TCCD parameters were assessed at both admission and discharge. The scales used were the Scandinavian stroke scale (SSS), Barthel Index (BI), modified Rankin Scale (mRS), FuglMeyer upper extremity scale (FMA-UE), modified motor assessment scale (mMAS) scores. TCCD parameters measured were MCA peak systolic, end diastolic, mean flow velocities (MFV), and index of symmetry ( $\mathrm{SI}$ ) and were correlated with clinical scores. Results Fourteen patients were recruited with median age of 56.5 years, median duration of stroke was 42.5 days. Mean flow velocities of affected and unaffected MCA were 46.2 and $50.7 \mathrm{~cm} / \mathrm{s}$, respectively. Flow velocities and SI did not change between the two assessments. There was significant improvement in clinical outcome scores at discharge. Significant correlation was observed for patient group with $\mathrm{SI}>0.9$ at admission with FMA-UE, SSS, and BI scores at discharge $(p<0.05)$.

Conclusion Flow velocity parameters did not change during in-patient rehabilitation. Patients with symmetric flow at admission had improved clinical outcomes measure scores at discharge. Thus SI can predict rehabilitation outcomes in stroke survivors. published online January 12,2022
DOI https://doi.org/

10.1055/s-0041-1742158. ISSN 0976-3147. (c) 2022. Association for Helping Neurosurgical Sick People. All rights reserved.

This is an open access article published by Thieme under the terms of the Creative Commons Attribution-NonDerivative-NonCommercial-License, permitting copying and reproduction so long as the original work is given appropriate credit. Contents may not be used for commercial purposes, or adapted, remixed, transformed or built upon. (https://creativecommons.org/ licenses/by-nc-nd/4.0/)

Thieme Medical and Scientific Publishers Pvt. Ltd., A-12, 2nd Floor, Sector 2, Noida-201301 UP, India 


\section{Introduction}

Conventional clinical predictors of stroke include age, risk factors, severity of stroke at onset, presence of proximal, and distal upper limb recovery in acute poststroke period, presence of aphasia at onset of stroke, and hemispatial neglect. ${ }^{1}$ Lesion size on magnetic resonance imaging (MRI) and the presence of subcortical strokes involving the corticospinal tracts are related with poor outcome. ${ }^{2}$

Coupling of blood flow to neuronal activity (neurovascular coupling) is detected with functional MRI (fMRI), transcranial Doppler, and functional near-infrared spectroscopy. ${ }^{3}$ Clinical predictors may lack the sensitivity to predict subtle changes of neuroplasticity and the commonly used neuroimaging tools may be unsuitable due to constraints in resource availability. ${ }^{3}$

Transcranial Doppler (TCD) and transcranial color-coded duplex (TCCD) sonography enable detection of intracranial vessels blood flow and are intuitively suited to study sequelae of cerebrovascular disease. ${ }^{4}$ Studies have shown good inter and intraobserver reliability of the techniques. ${ }^{4,5}$ TCCD is inexpensive in comparison to other neuroimaging tools, noninvasive, portable, and readily available in clinical practice. The present study was aimed to evaluate findings of TCCD sonography for middle cerebral artery (MCA) territory infarcts in patients undergoing stroke rehabilitation and to correlate TCCD sonography findings with patients' clinical outcome measures. This study used TCCD and not TCD which is the most commonly used tool to obtain intracranial flow velocities. The advantages of TCD over TCCD include higher sensitivity for blood flow detection due to dedicated Doppler function, ability to conduct autoregulation studies, and the availability of normative data for velocities detected with TCD. While we describe absolute velocities obtained on TCCD, we also used the symmetry index which can circumvent reliance on absolute velocity values.

\section{Materials and Methods}

The study population included male and female patients aged between 18 and 65 years admitted in the rehabilitation wards within 6 months of onset of a first MCA infarct. The patients with recurrent stroke, venous stroke, intracerebral or subarachnoid hemorrhage, past history of lesion localized to the central nervous system, and those with global aphasia were not included. Institute Ethical Committee clearance was obtained for the study.

\section{Study Tools}

1. Clinical outcome measures used at the time of admission and discharge were as follows:

- Fugl-Meyer upper extremity scale (FMA-UE): we limited the use of this scale to the motor subset in the present study, with a maximum of 66 points. ${ }^{6}$

- Scandinavian stroke scale (SSS): it is a nine-item scale with a maximum score of 58 and has been used to measure disability or evaluate prognosis of stroke patients. $^{7}$

- Modified motor assessment scale (mMAS): it is a measure of upper, lower limb, and trunk function and assesses motor recovery. It covers six domains and is scored on a 7point scale from 0-6 with a total score of 48. 8

- The modified Rankin scale (mRS): it measures the degree of disability or dependence in the daily activities of people who have suffered stroke. ${ }^{8,9}$

- The Barthel index (BI): it is an ordinal scale used to measure performance in activities of daily living (ADL), with higher scores signifying greater degree of independence. ${ }^{8,9}$

2. TCCD sonographic assessment: parameters measured were listed below:

- Peak systolic velocity of MCA.

- End diastolic velocity of MCA.

- Mean flow velocity calculated using the formula: MCA mean flow velocity $=1 / 3$ MCA peak systolic velocity $+2 / 3$ MCA end diastolic velocity.

- Index of symmetry (SI) calculated as the ratio of mean flow velocity of affected hemisphere and mean flow velocity of unaffected hemisphere.

\section{Methods}

The TCCD scan was performed on the day of admission and at time of discharge from rehabilitation unit. The patient was made to lie supine for 5 minutes. A low-frequency $2.5-\mathrm{MHz}$ transducer was used. The probe was placed along the temporal window (along the orbitomeatal line) and B-mode imaging was used to identify gross intracranial landmarks. Color flow imaging was used to identify intracranial vessels. The MCA is identified as the vessel with flow toward the probe and emerging from the internal carotid artery, at a depth of 4 to $6 \mathrm{~cm}$ from the surface. Doppler mode was used to determine peak systolic velocity and end diastolic velocity. Measurements were made for both hemispheres sequentially.

All the patients were given customized rehabilitation sessions. A rehabilitation session consisted of physical and occupational therapy, speech therapy, and neuropsychology sessions.

\section{Statistical Analysis}

Statistical analysis was performed with Stata software. Comparison of clinical outcome measures and TCCD parameters at admission and discharge was done with Wilcoxon's matched-pair signed-rank test. For comparison between two groups of patients, that is, those with $\mathrm{SI} \leq 0.9$ and those with $\mathrm{SI}<0.9$, Wilcoxon's rank-sum test (Mann-Whitney two-sample statistics) was applied.

\section{Results}

A total of 17 patients satisfied inclusion criteria. Of these, three had inadequate bone window, and thus 14 patients were included. There were 11 men and median age of 
Table 1 Comparison of TCCD parameters and clinical outcome measures at admission and at discharge

\begin{tabular}{|l|l|l|l|}
\hline Variable & $\begin{array}{l}\text { Scores at admission } \\
\text { Median (IQR) }\end{array}$ & $\begin{array}{l}\text { Scores at discharge } \\
\text { Median (IQR) }\end{array}$ & -Value $^{\mathbf{a}}$ \\
\hline Age (y) & $56.5(45,62)$ & - & - \\
\hline Duration (d) & $42.5(21,65)$ & - & - \\
\hline TCCD parameters & & & \\
\hline MFVa(cm/s) & $43.67(37.65,54.71)$ & $45.04(36.33,54.22)$ & 0.7901 \\
\hline MFVu (cm/s) & $46.95(36.42,56.86)$ & $51.85(37.80,55.16)$ & 0.424 \\
\hline MFVa/MFVu & $0.92(0.83,0.96)$ & $0.96(0.93,0.99)$ & 0.267 \\
\hline Clinical outcome measures & & & \\
\hline SSS & $23.5(17.5,30)$ & $43(33,45)$ & 0.0001 \\
\hline BI & $20(15,35)$ & $60(55,65)$ & 0.0001 \\
\hline FMA-UE & $7(4,9)$ & $18.5(9,22)$ & 0.001 \\
\hline mMAS & $11.5(7,15)$ & $22.5(17,25)$ & 0.001 \\
\hline mRS & $4(3.25,4)$ & $3(2,3)$ & 0.0005 \\
\hline
\end{tabular}

Abbreviations: a, affected hemisphere; BI, the Barthel index; FMA-UE, Fugl-Meyer assessment upper extremity; MFV, mean flow velocity; mMAS, the modified motor activity score; mRS, modified Rankin scale; SSS, Scandinavian stroke scale; TCDD, transcranial color-coded duplex sonography; u, unaffected hemisphere.

aWilcoxon's matched-pairs signed-ranks test.

Table 2 Comparison of clinical outcome measure scores at discharge and between two groups (group 1: SI $\leq 0.9$, group 2: SI $<0.9$ at admission)

\begin{tabular}{|l|l|l|l|}
\hline Clinical outcome measure & $\begin{array}{l}\text { Group 1 (SI } \leq \mathbf{0 . 9}[\boldsymbol{n}=\mathbf{9}]) \\
\text { Median (IQR) }\end{array}$ & $\begin{array}{l}\text { Group 2 (SI }<\mathbf{0 . 9}[\boldsymbol{n}=\mathbf{5}]) \\
\text { Median (IQR) }\end{array}$ & $p$-Value \\
\hline SSS & $45(43,47)$ & $32(31,33)$ & 0.0026 \\
\hline BI & $55(60,70)$ & $55(45,55)$ & 0.0023 \\
\hline FMA-UE & $22(19,23)$ & $7(7,9)$ & 0.0026 \\
\hline mMAS & $23(22,29)$ & $16(13,20)$ & 0.0529 \\
\hline mRS & $2(2,3)$ & $3(3,3)$ & 0.0452 \\
\hline
\end{tabular}

Abbreviations: BI, the Barthel index; FMA-UE, Fugl-Meyer assessment upper extremity; IQR, interquartile range; mMAS, modified motor activity score; mRS, the modified Rankin scale; SI, index of symmetry; SSS, Scandinavian stroke scale.

aWilcoxon's rank-sum test (Mann-Whitney two-sample statistic).

patients was 56.5 years. The study had 8 patients with left MCA territory infarcts and 11 patients had cortical strokes. Nine patients had SI value greater than 0.9 .

- Table 1 shows the demographic data, TCCD parameters, and clinical outcome measures at admission and at discharge. - Table 2 shows the comparison of scores at discharge between two groups of patients, group 1 with SI value of $\leq 0.9$ at admission, and group 2 with $\mathrm{SI}<0.9$ at admission.

\section{Discussion}

We recorded MCA flow velocity with TCCD sonography at admission and discharge from rehabilitation unit. Three (17.6\%) out of a total of 17 recruited patients had poor acoustic window. This is comparable with published evidence which puts the percentage of inadequate acoustic window between 10 and $15 \%{ }^{10}$ Median duration of symptoms at admission was 42.5 days which indicates subacute stroke. This is the time period where the effects of plasticity and rehabilitation interventions can positively change patient outcomes. Eight patients (57\%) had left hemisphere infarcts while remaining had right hemispheric.

The change in clinical outcome measures between admission and discharge is statistically significant for BI, FMA-UE, SSS, mMAS, and mRS scale scores. This can be considered to be the mixed effect of natural progression of recovery (plasticity) and in-patient rehabilitation program. Mean flow velocities of patients were in the normative range. ${ }^{11}$ However, normative data of MCA mean flow velocities is predominantly from TCD studies. TCD probes are more sensitive to pick up flow signals; however, TCCD is advantageous in terms of the ability to use angle-correction on detected velocities. Angle correction function in TCCD changes mean flow velocity values by up to $45.9 \%{ }^{12}$ In our study, we did not use angle correction to align our methods with TCD studies.

There were no statistically significant difference in mean flow velocities between admission and discharge which is consistent with findings by an earlier study. Occluded MCAs 
have also been shown to recanalize after 2 weeks of stroke. ${ }^{13}$ Hence, flow velocities may not be ideal parameters to glean information on poststroke outcomes for study populations in subacute and chronic settings. To address this issue, we incorporated an index of symmetric flow. In published literature, the MCA asymmetry indices are described with the use of terms left and right. We did not utilize the standard MCA laterality index due to a lack of clarity in the definition of the index with respect to laterality. ${ }^{14}$ Hence we used a ratio (SI) obtained by dividing the mean flow velocity of MCA of affected hemisphere with MCA mean flow velocity of unaffected side. This is similar to the approach followed by Brint et al. ${ }^{15}$ There was no statistically significant change in the SI between the two time points in our study.

We observed a positive correlation between the SI at admission and clinical outcome measures such as the BI, SSS, and FMA-UE at discharge. This finding may suggest that symmetry in flow velocities between the affected and unaffected hemisphere at admission is predictive of functional outcome after rehabilitation.

A study by Treger et al in 2005 reported that greater flow velocities of contralesional hemisphere were associated with poor outcome at discharge. ${ }^{16}$ Same group reported contrasting findings in a study in 2010 where blood flow in the contralesional hemisphere was associated with functional improvement. ${ }^{17}$ Brint et al in a TCD study observed that the risk of stroke was greater with asymmetry of flow. ${ }^{15}$ Han et al described a novel index of asymmetry incorporating flow velocity and pulsatility index to predict stroke outcomes. ${ }^{14}$ The fMRI studies indicate that symmetric blood flow between the two hemispheres and return of activity to affected cortex is associated with improved outcomes for stroke patients and is a marker of recovery and rehabilitation effect. ${ }^{18}$ Thus the available literature on studies recording blood flow indicates that symmetric activity of two hemispheres is associated with better functional outcome. Our study demonstrates this observation using TCCD and shows that TCCD can be used as a cost-effective method to predict outcomes of rehabilitation. TCD studies done in the acute setting have shown that MCA mean flow velocities less than $30 \mathrm{~cm} / \mathrm{s}$ relate to poor functional outcome. Absent flow in the affected MCA is predictive of mortality following stroke. ${ }^{19}$ In addition to prognostication, TCCD/TCD can be used as a functional imaging tool and an assessment modality for the effect of interventions. For such applications, MCA velocities are measured during the performance of a motor or cognitive task. Such studies are based on the phenomenon of neuromuscular coupling. ${ }^{3}$ A study by Béné et al measured MCA flow velocities after simulating mirror therapy in healthy patients. They reported significant increase in mean flow velocity after the task TCD. ${ }^{20}$

Despite promising uses, not many TCD studies have been conducted in rehabilitation setups. It is a specialized tool and may not be accessible to rehabilitation units. In such a scenario, TCCD might be more feasible in centers where phased array probes are available. However, the learning curve, time taken to perform TCCD scans, interest, and requisite training in the technique are some of the important challenges in conducting TCCD studies in rehabilitation units.

\section{Conclusion}

The use of TCCD sonography in a rehabilitation setting with stroke survivors is feasible. All patients showed functional improvements. Flow velocities did not change over the course of in-patient rehabilitation. There was a positive correlation between the SI and functional outcome scores. This finding gives direction toward the potential use of TCCD as an additional prognostic tool for stroke survivors.

\section{Funding \\ None.}

\section{Conflict of Interest}

None declared.

\section{References}

1 Douiri A, Grace J, Sarker SJ, et al. Patient-specific prediction of functional recovery after stroke. Int J Stroke 2017;12(05):539-548

2 Crosson B, Ford A, McGregor KM, et al. Functional imaging and related techniques: an introduction for rehabilitation researchers. J Rehabil Res Dev 2010;47(02):vii-xxxiv

3 Phillips AA, Chan FH, Zheng MMZ, Krassioukov AV, Ainslie PN. Neurovascular coupling in humans: physiology, methodological advances and clinical implications. J Cereb Blood Flow Metab 2016;36(04):647-664

4 D'Andrea A, Conte M, Cavallaro M, et al. Transcranial Doppler ultrasonography: from methodology to major clinical applications. World J Cardiol 2016;8(07):383-400

5 Dupont G, Burnol L, Jospe R, et al. Transcranial color duplex ultrasound: a reliable tool for cerebral hemodynamic assessment in brain injuries. J Neurosurg Anesthesiol 2016;28(02):159-163

6 Lundquist CB, Maribo T. The Fugl-Meyer assessment of the upper extremity: reliability, responsiveness and validity of the Danish version. Disabil Rehabil 2017;39(09):934-939

7 Askim T, Bernhardt J, Churilov L, Indredavik B. The Scandinavian Stroke Scale is equally as good as The National Institutes of Health Stroke Scale in identifying 3-month outcome. J Rehabil Med 2016; 48(10):909-912

8 Loewen SC, Anderson BA. Reliability of the modified motor assessment scale and the Barthel index. Phys Ther 1988;68(07): 1077-1081

9 Dromerick AW, Edwards DF, Diringer MN. Sensitivity to changes in disability after stroke: a comparison of four scales useful in clinical trials. J Rehabil Res Dev 2003;40(01):1-8

10 Purkayastha S, Sorond F. Transcranial Doppler ultrasound: technique and application. Semin Neurol 2012;32(04):411-420

11 Ringelstein EB, Kahlscheuer B, Niggemeyer E, Otis SM. Transcranial Doppler sonography: anatomical landmarks and normal velocity values. Ultrasound Med Biol 1990;16(08):745-761

12 Krejza J, Mariak Z, Babikian VL. Importance of angle correction in the measurement of blood flow velocity with transcranial Doppler sonography. AJNR Am J Neuroradiol 2001;22(09):1743-1747

13 Alexandrov AV, Bladin CF, Norris JW. Intracranial blood flow velocities in acute ischemic stroke. Stroke 1994;25(07):1378-1383

14 Han M, Kim YD, Park HJ, et al. Prediction of functional outcome using the novel asymmetric middle cerebral artery index in cryptogenic stroke patients. PLoS One 2019;14(01):e0208918

15 Brint SU, Al-Khalidi HR, Vatel B, Hier DB. MCA flow asymmetry is a marker for cerebrovascular disease. Neurol Res 1996;18(02): $163-167$ 
16 Treger I, Streifler JY, Ring H. The relationship between mean flow velocity and functional and neurologic parameters of ischemic stroke patients undergoing rehabilitation. Arch Phys Med Rehabil 2005;86(03):427-430

17 Treger I, Aidinof L, Lutsky L, Kalichman L. Mean flow velocity in the middle cerebral artery is associated with rehabilitation success in ischemic stroke patients. Arch Phys Med Rehabil 2010;91(11):1737-1740

18 Johansen-Berg H, Dawes H, Guy C, Smith SM, Wade DT, Matthews PM. Correlation between motor improvements and altered fMRI activity after rehabilitative therapy. Brain 2002;125(pt. 12):2731-2742

19 Sarkar S, Ghosh S, Ghosh SK, Collier A. Role of transcranial Doppler ultrasonography in stroke. Postgrad Med J 2007;83(985): 683-689

20 Béné R, Lovrencić-Huzjan A, Azman D, et al. Blood flow velocity in middle cerebral artery during visuo-motor tasks using a mirror: a transcranial Doppler study. Acta Clin Croat 2009;48 (03):305-310 